\title{
A new approach to the long-term reconstruction of the solar irradiance leads to large historical solar forcing ${ }^{\star}$
}

\author{
A. I. Shapiro ${ }^{1}$, W. Schmutz ${ }^{1}$, E. Rozanov ${ }^{1,2}$, M. Schoell ${ }^{1,3}$, M. Haberreiter ${ }^{1}$, A. V. Shapiro ${ }^{1,2}$, and S. Nyeki ${ }^{1}$ \\ ${ }^{1}$ Physikalisch-Meteorologishes Observatorium Davos, World Radiation Center, 7260 Davos Dorf, Switzerland \\ e-mail: alexander.shapiro@pmodwrc.ch \\ 2 Institute for Atmospheric and Climate science ETH, Zurich, Switzerland \\ ${ }^{3}$ Institute for Astronomy ETH, Zurich, Switzerland
}

Received 19 November 2010 / Accepted 22 February 2011

\begin{abstract}
Context. The variable Sun is the most likely candidate for the natural forcing of past climate changes on time scales of 50 to 1000 years. Evidence for this understanding is that the terrestrial climate correlates positively with the solar activity. During the past 10000 years, the Sun has experienced the substantial variations in activity and there have been numerous attempts to reconstruct solar irradiance. While there is general agreement on how solar forcing varied during the last several hundred years - all reconstructions are proportional to the solar activity - there is scientific controversy on the magnitude of solar forcing.

Aims. We present a reconstruction of the total and spectral solar irradiance covering $130 \mathrm{~nm}-10 \mu \mathrm{m}$ from 1610 to the present with an annual resolution and for the Holocene with a 22-year resolution.

Methods. We assume that the minimum state of the quiet Sun in time corresponds to the observed quietest area on the present Sun. Then we use available long-term proxies of the solar activity, which are ${ }^{10} \mathrm{Be}$ isotope concentrations in ice cores and 22-year smoothed neutron monitor data, to interpolate between the present quiet Sun and the minimum state of the quiet Sun. This determines the longterm trend in the solar variability, which is then superposed with the 11-year activity cycle calculated from the sunspot number. The time-dependent solar spectral irradiance from about $7000 \mathrm{BC}$ to the present is then derived using a state-of-the-art radiation code.

Results. We derive a total and spectral solar irradiance that was substantially lower during the Maunder minimum than the one observed today. The difference is remarkably larger than other estimations published in the recent literature. The magnitude of the solar UV variability, which indirectly affects the climate, is also found to exceed previous estimates. We discuss in detail the assumptions that lead us to this conclusion.
\end{abstract}

Key words. solar-terrestrial relations - Sun: UV radiation - Sun: atmosphere - radiative transfer - line: formation Sun: surface magnetism

\section{Introduction}

The Sun is a variable star whose activity varies over time-scales ranging from minutes to millennia. Over the last thirty years the solar irradiance was measured by numerous space missions. Measurements of the total solar irradiance (TSI) became available with the launch of the NIMBUS 7 mission in 1978 (Hoyt et al. 1992). Since then the TSI was measured by several consecutive instruments. Each of them suffered from degradation and individual systematic effects, which renders the direct comparison of the measurements impossible. Three TSI composites based on the available data were constructed by three groups: PMOD (Fröhlich 2006), ACRIM (Willson \& Mordvinov 2003), and IRMB (Dewitte et al. 2004). These composites give quite different values of the TSI, especially before 1980 and during the so-called ACRIM gap between June 1989 (end of the ACRIM I observations) and October 1991 (beginning of the ACRIM II observations). The most striking detail is the increase of the TSI between the minima 1986 and 1996 in the ACRIM composite and the absence of this increase in the PMOD and IRMB composites. Although significant progress was made during the last few years and the increase of the TSI in the ACRIM composite

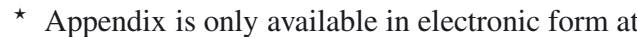
http://www . aanda. org was strongly criticized (Fröhlich 2009; Krivova et al. 2009a), the question of the construction of the unique self-consistent TSI composite for the satellite epoch is still open.

Measurements of the spectral solar irradiance (SSI) are even more difficult, and instrumental problems prevent the construction of essential composites (Krivova et al. 2011; Domingo et al. 2009). Recently (Krivova et al. 2009b) the theoretical SATIRE (Spectral And Total Irradiance REconstruction) (Krivova et al. 2003; Krivova \& Solanki 2008) model and SUSIM (Solar Ultraviolet Spectral Irradiance Monitor) measurements were used to reconstruct the solar UV irradiance back to 1974.

Taking into account the problems of the reconstruction of the TSI and SSI during the recent period of satellite observations and combining it with the fact that the solar dynamo, which is believed to drive all activity manifestations, is still not fully understood (Charbonneau 2010), one recognizes the difficulty of reconstructing the TSI and SSI into the past, when no direct measurements were available.

Long-term changes in solar irradiance were suspected as early as the mid-nineteenth century (Smyth 1855). One of the first quantitative estimates of its magnitude as well as past solar irradiance reconstructions was obtained by using the observations of solar-like stars (Lean et al. 1995). It was concluded that the TSI during the Maunder minimum was about $3-4 \mathrm{~W} / \mathrm{m}^{2}$ less 
than at present, which translates into a solar radiative forcing ${ }^{1}$ $\Delta F_{\mathrm{P}-\mathrm{M}} \sim 0.5-0.7 \mathrm{~W} / \mathrm{m}^{2}$. However, these results were not confirmed by extended surveys of solar-like stars and are no longer considered to be correct (see Hall \& Lockwood 2004). Recent reconstructions based on the magnetic field surface distribution (Wang et al. 2005; Krivova et al. 2007) and on an extrapolation of the assumed correlation between the TSI and the open magnetic flux (see definition in Lockwood et al. 1999) during the last three minima (Steinhilber et al. 2009; Fröhlich 2009) resulted in a low solar forcing value within the range $\Delta F_{\mathrm{P}-\mathrm{M}} \approx$ $0.1-0.2 \mathrm{~W} / \mathrm{m}^{2}$.

\section{Effects of solar radiative forcing on the climate}

Variations on time-scales of up to the 27-day rotational period have an important influence on space weather, but not on the terrestrial climate. The effects of the 11-year solar cycle are clearly detected in the atmosphere and are widely discussed in the literature (e.g., Egorova et al. 2004; Haigh 2007), while the imprints from variations on longer time-scales are more subtle (Gray et al. 2010). An analysis of the historical data suggests a strong correlation between the solar activity and the natural climate variations on centennial time-scales, such as the colder climate during the Maunder (about 1650-1700 AD) and Dalton (about 1800-1820 AD) minima as well as climate warming during the steady increase in solar activity in the first half of the twentieth-century (Siscoe 1978; Hoyt \& Schatten 1997; Solomon et al. 2007; Gray et al. 2010). Numerous attempts to confirm these correlations based on different climate models have shown that it is only possible if either the applied perturbations of direct solar radiative forcing are large (consistent with a direct solar radiative forcing from the present to Maunder minimum $\Delta F_{\mathrm{P}-\mathrm{M}} \sim 0.6-0.8 \mathrm{~W} / \mathrm{m}^{2}$ ) or if the amplification of a weak direct solar forcing is substantial. Because the majority of recent $\Delta F_{\mathrm{P}-\mathrm{M}}$ estimates (see Sect. 1) are only in the range $0.1-0.2 \mathrm{~W} / \mathrm{m}^{2}$, and amplification processes have not been identified, the role of the solar forcing in the natural climate change remains highly uncertain (Solomon et al. 2007). In this paper we show that the solar forcing may be significantly larger than reported in the recent publications.

\section{Methods}

We present a new alternative technique, which avoids calibration of our model with the presently observed TSI variations and an extrapolation into the past. We assert that the amount of magnetic energy that remains present (de Wijn et al. 2009) at the surface of a spotless (i.e. quiet) Sun is the main driver of solar irradiance variability on centennial time scales. The main concept of our technique is to determine the level of the magnetically enhanced contribution to the irradiance of the present quiet Sun. Then, for the reconstruction into the past, this magnetically enhanced component has to be scaled with the proxies for the quiet Sun activity. However, a proxy for the long-term activity of the quiet Sun does not yet exist. A good candidate for such a proxy are the small-scale turbulent magnetic fields that are accessed with the Hanle effect (Stenflo 1982). However, the consecutive measurements of these fields are limited to the last few years (Kleint et al. 2010). Therefore, we assume that the existing proxies of the solar activity averaged over a two solar cycles period

\footnotetext{
1 Solar radiative forcing is a direct energy source to the Earth and is related to the change in TSI by $\Delta F=\Delta \mathrm{TSI} \cdot(1-A) / 4$, where $A$ is the Earth's albedo.
}

can also describe the activity of the quiet Sun. The averaging of proxies allows sufficient time for the magnetic components to decay into the quiet network and then to even smaller magnetic features because the decay process can last up to several years (Solanki et al. 2000). In other words, we set the smallscale activity (which defines the fractional contributions of quiet Sun components) to be proportional to the large-scale activity. We clearly state that this proposition is an assumption, which is however in line with the high-resolution observations of the present Sun. The large-scale structure caused by strong magnetically active features is repeated on a smaller scale in less active regions, and even in the apparently quietest areas there is still a mosaic of regions of different magnetic field strengths, reminiscent of a fractal structure (de Wijn et al. 2009).

Hence, the time-dependent irradiance $I_{\text {quiet }}(\lambda, t)$ of the quiet Sun in our reconstruction can be calculated as

$\frac{I_{\text {quiet }}(\lambda, t)-I_{\text {min.state }}(\lambda)}{\langle\text { Proxy }\rangle_{22}(t)}=\frac{I_{\text {quiet }}\left(\lambda, t_{0}\right)-I_{\text {min.state }}(\lambda)}{\langle\text { Proxy }\rangle_{22}\left(t_{0}\right)}$,

where $I_{\text {quiet }}(\lambda, t)$ is the time-dependent irradiance of the quiet Sun and $t_{0}$ denotes a reference time. Proxy $y_{22}$ is the value of the proxy for the solar activity averaged over a 22 -year period. The 22-year period was chosen because the cosmogenic isotope data used for the reconstruction (see below) are available as 22-year averaged data (Steinhilber et al. 2008). We have set 1996 as the reference year, i.e. $I_{\text {quiet }}\left(\lambda, t_{0}\right)$ is the irradiance of the quiet Sun as observed during the 1996 minimum. Let us notice that the quiet Sun irradiance was roughly constant for the last three cycles (see below). Therefore, the solar spectrum during the 1996 minimum is a good representation of the present quiet Sun spectrum. $I_{\text {min.state }}(\lambda)$ is the irradiance of an absolute minimum state of the Sun, with a minimum of remaining magnetic flux emerging on the solar surface. Thus, the $I_{\text {quiet }}\left(\lambda, t_{0}\right)-I_{\text {min.state }}(\lambda)$ term can be considered as the enhancement level of the present quiet Sun with respect to the Sun in its most inactive state.

The prominent, readily observable active regions on the Sun also contribute to the variability in irradiance. Thus, the full solar variability is described by

$I(\lambda, t) \equiv I_{\text {quiet }}(\lambda, t)+I_{\text {active }}(\lambda, t)$,

where $I_{\text {active }}(\lambda, t)$ is the contribution to solar irradiance from active regions, e.g. sunspots, plages, and network. $I_{\text {active }}(\lambda, t)$ is calculated following the approach by Krivova et al. (2003; see also Appendix A) and, because the calculations are made with annular resolution, is proportional to the sunspot number. The group sunspot number used in our reconstruction is taken from the NOAA data center and is described in Hoyt \& Schatten (1998).

The term $I_{\text {active }}(\lambda, t)$ in Eq. (2) describes the cyclic component of solar variability caused by the 11-year activity cycle, while the slower long-term changes owing to the evolution of the small-scale magnetic flux are given by Eq. (1). The $I_{\text {active }}(\lambda, t)$ term can only be calculated for the time when a sunspot number is available and therefore our reconstruction has a 22-year resolution for the Holocene and an annual resolution from 1610 to present.

The choice of the model for the minimum state of the Sun is a crucial point in our technique because it defines the amplitude of the reconstructed solar irradiance variability. Observations of the Sun with relatively high spatial resolution show that the present quiet Sun is still highly inhomogeneous, even though it appears to be spotless. Measurements obtained with the Harvard spectroheliometer aboard Skylab were used to derive the brightness components of the quiet Sun and to construct 
the corresponding semi-empirical solar atmosphere structures (Vernazza et al. 1981; Fontenla et al. 1999). The darkest regions with the least amount of magnetic flux correspond to the faint supergranule cell interior (component A). This component comes closest to describing the most inactive state of the Sun. Therefore, in our approach we set $I_{\text {min.state }}(\lambda) \equiv I_{\mathrm{A}}(\lambda)$. Thus, the basic magnitude of the solar irradiance variations is given by the difference between the irradiance of the present quiet Sun (composed from a distribution of brightness components defined in Appendix A) and the irradiance from component A (see Eq. (1)).

For the reconstruction into the past this amplitude is scaled with proxies for the solar activity. Two proxies are available for the reconstruction: group sunspot number, which is available from the present to $1610 \mathrm{AD}$, and the solar modulation potential, which extends back to circa $7300 \mathrm{BC}$. The latter is a measure of the heliospheric shielding from cosmic rays derived from the analysis of cosmogenic isotope abundances in tree rings or ice cores, and is available with a time resolution of 2-3 solar cycles (Steinhilber et al. 2008). Although the sunspot number dropped to zero for a long time during the Maunder minimum, the solar cycle was uninterrupted (Beer et al. 1998; Usoskin et al. 2001) and the modulation potential did not fall to zero. Hence, a reconstruction based solely on the sunspot number may underestimate the solar activity during the Maunder minimum. Therefore, we used in our reconstruction the solar modulation potential to calculate the long-term variations and the sunspot number to superpose them with the 11-year cycle variations (see Appendix A).

The modulation potential used in the calculations is based on the composite of data determined from the cosmogenic isotope records of ${ }^{10} \mathrm{Be}$ and a neutron monitor. ${ }^{10} \mathrm{Be}$ data are available up to about 1970 (McCracken et al. 2004) and the neutron monitor data, which are used to calculate the current solar modulation potential, are available since the 1950s. Three different datasets of the ${ }^{10} \mathrm{Be}$ data were used: measurements at DYE 3, Greenland, and the South Pole provided by McCracken et al. (2004) for the reconstruction back to the Maunder minimum, and measurements from the Greenland Ice core Project provided by Vonmoos et al. (2006) for the reconstruction back to $7300 \mathrm{BC}$. The neutron monitor data were provided by Usoskin et al. (2005). Although they are available with a monthly resolution, we calculated the 22-year mean of the neutron monitor to homogenize the data sets and because only an averaged modulation potential can be used as a proxy for the quiet Sun activity. The transition from ${ }^{10} \mathrm{Be}$ to neutron monitor data is shown in the lower panel of Fig. 1. Before 1952 the composites are based on the ${ }^{10} \mathrm{Be}$ data (cyan based on DYE 3, and red on South Pole records, both with 22-year resolution). The values between 1952 and 1998 are obtained by linearly interpolating the following consecutive data points: two blue crosses at 1952 (the last data points used from the ${ }^{10}$ Be records), green crosses at 1976 and 1998 (22-year averages of the neutron monitor data). The values after 1998 require knowledge of the next 22-year average (between 2010 and 2031) of the modulation potential. We assume this average to be $92 \%$ of the previous average (between 1988 and 2009), which allows us to reproduce the observed TSI minimum in 2008. The datasets mentioned above were derived using different assumptions of the local interstellar spectra (LIS). To homogenize the data we converted the modulation potential from McCracken et al. (2004) and Usoskin et al. (2005) to LIS by Castagnoli \& Lal (1980), which is used in Vonmoos et al. (2006). For conversion we applied a method suggested by Steinhilber et al. (2008). Herbst et al. (2010) analyzed the dependency of the modulation potential on the applied LIS models and showed that the negative values of the modulation potential in the Vonmoos et al. (2006)
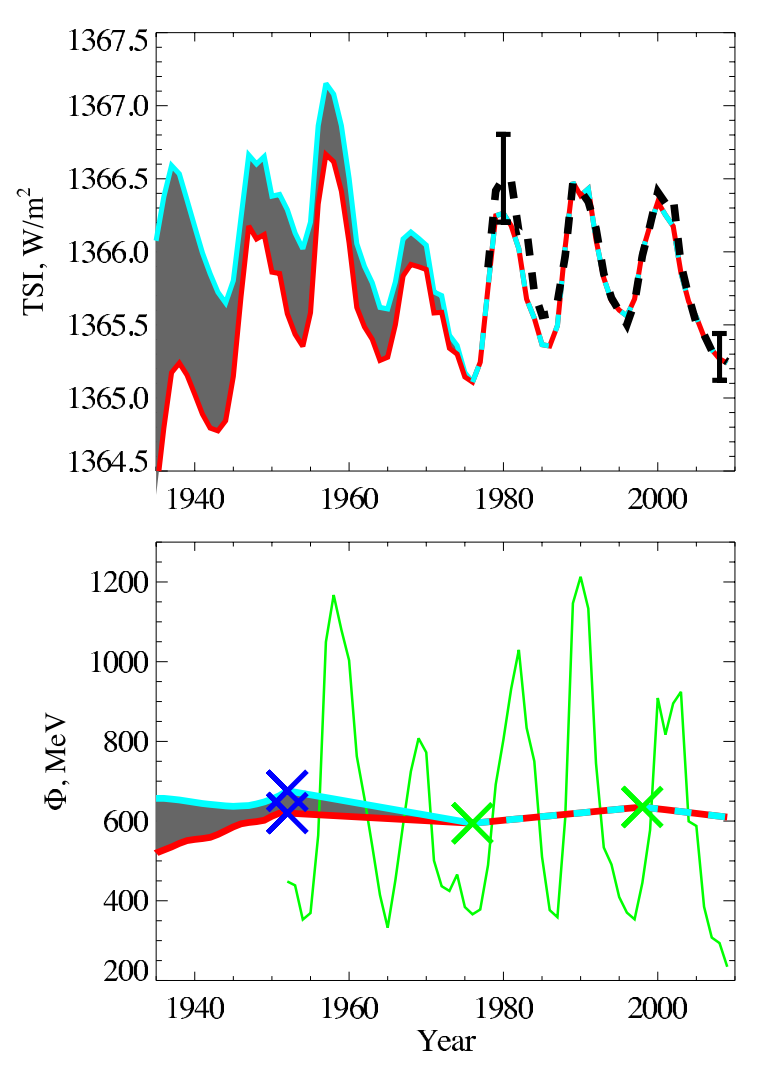

Fig. 1. Modulation potential and TSI reconstruction for the last 70 years. Lower panel: yearly averaged neutron monitor data (green) and two modulation potential composites (red and cyan curves, see the discussion in the text). Upper panel: TSI reconstructions based on the two modulation potential composites (red and cyan curves). The black dashed line is the observed TSI from the PMOD composite. The error bar for 1980 corresponds to $1 / 4$ the difference between two published TSI composites, and the error for 2008 is taken from Fröhlich (2009). The reconstructed TSI curves are normalized to the 1996 minimum and the gray-shaded region indicates the intrinsic uncertainty caused by differences in the modulation potential data.

data can be corrected with another LIS model. The solar forcing in our reconstruction is determined by the relative values of the modulation potential, which means that change to a different LIS model will introduce only several percents of correction, which is much less than the estimated accuracy of the reconstruction. In our dataset the value of the reference modulation potential $\langle\text { Proxy }\rangle_{22}\left(t_{0}\right)$ (see Eq. (1)) is equal to $631 \mathrm{MeV}$. The relative error is estimated to be less than $10 \%$ (Usoskin et al. 2005).

Synthetic solar spectra are calculated with a state-of-the-art radiative transfer code (Haberreiter et al. 2008; Shapiro et al. 2010). We discuss the calculations in more detail in Appendix A, where in addition we show that the concept of the quiet Sun activity scaling can also be expressed in terms of varying fractional contributions from different components of the quiet Sun.

\section{Results and discussion}

In the upper panels of Figs. 1 and 2 we present the TSI reconstructions, which are obtained after the integration of Eqs. (1) and (2) over the wavelengths and normalization of the quiet Sun value for the reference year 1996 to $1365.5 \mathrm{~W} / \mathrm{m}^{2}$. Because the sunspot number is only available since $1610 \mathrm{AD}$, the reconstruction of the full solar cycle variability with an annual 

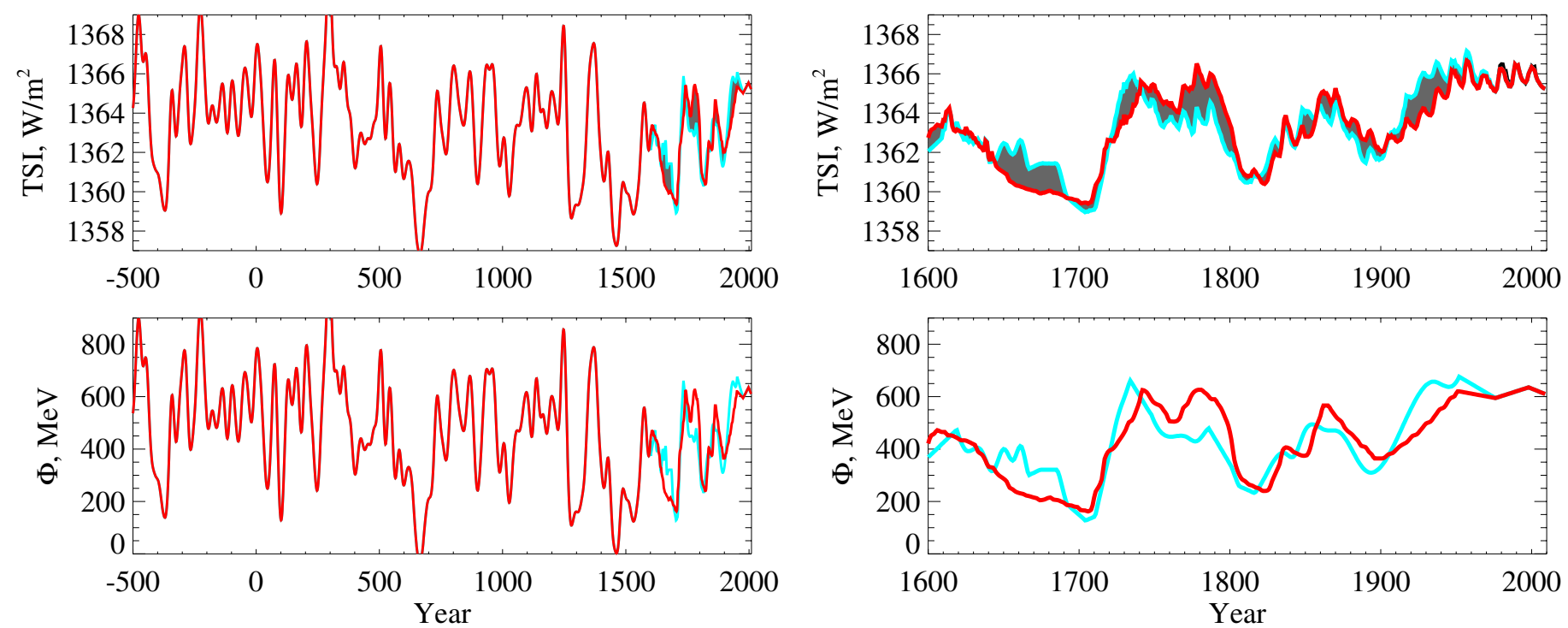

Fig. 2. Modulation potential (lower panel) and TSI reconstructions (upper panel) for the last 2500 years. Data before 1600 AD are based on the modulation potential derived from ${ }^{10} \mathrm{Be}$ records from the Greenland Ice core Project (red curves). Data since 1600 AD are based on the two composites shown in Fig. 1 (red and cyan curves). The gray-shaded area indicates the intrinsic uncertainty.

resolution extends back only 400 years. Both reconstructions in the right-hand panel of Fig. 2 are based on the ${ }^{10}$ Be data sets mentioned above. The difference in the reconstructions allows the error originating from the uncertainties in the proxy data to be estimated (20-50\% in the solar forcing value, depending on the year). This is large, but still significantly less than the change in irradiance between the present and the Maunder minimum. Both reconstructions suggest a significant increase in the TSI during the first half of the twentieth-century as well as a low solar irradiance during the Maunder and Dalton minima. The difference between the current and the reconstructed TSI during the Maunder minimum is about $6 \pm 3 \mathrm{~W} / \mathrm{m}^{2}$ (equivalent to a solar forcing of $\Delta F_{\mathrm{P}-\mathrm{M}} \sim 1.0 \pm 0.5 \mathrm{~W} / \mathrm{m}^{2}$ ), which is substantially larger than recent estimates (see Sect. 1). Because our technique uses 22-year means of the solar modulation potential, our approach cannot be tested with the last, unusual solar minimum in 2008. To reproduce the current minimum as shown in Fig. 1, we adopted a value of $584 \mathrm{MeV}$ for the future 22-year average in 2020 (which is $92 \%$ of the 22-year average for 1988-2009).

The reconstruction before $1500 \mathrm{AD}$ in the left-hand panel of Fig. 2 (which stops for clarity at $500 \mathrm{BC}$ ) is based on the ${ }^{10} \mathrm{Be}$ records from the Greenland Ice core Project (Vonmoos et al. 2006). The modulation potential during the Maunder minimum is about 3-4 times less than at present, but not zero. However, it has decreased to zero several times in the past, and the corresponding TSI was even smaller than during the Maunder minimum. There were also several periods when the modulation potential, and hence the TSI, were higher than the present value. The reconstruction back to $7000 \mathrm{BC}$ is presented in Fig. 3. The choice of model A introduces an uncertainty of the order of $30 \%$, which is estimated by comparing model A to other possible candidates for the minimum state of the quiet Sun, e.g., model B from Vernazza et al. (1981). Combining this with the uncertainties of the proxy data outlined in Fig. 2 we can roughly estimate the uncertainty of our solar forcing value to be $50 \%$. In additional to the ${ }^{10} \mathrm{Be}$-based data of the solar activity there are several ${ }^{14} \mathrm{C}$-based datasets (e.g., Solanki et al. 2004; Vonmoos et al. 2006; Muscheler et al. 2007; Usoskin 2008). An employment of these datasets will lead to somewhat different values of the solar variability, which is, however, covered by our rough order of magnitude estimate of the overall uncertainty of the reconstruction.

Our TSI reconstructions give a value of $\sim 1 \mathrm{~W} / \mathrm{m}^{2}$ per decade for the period 1900-1950. The Smithsonian Astrophysical Observatory (SAO) has a 32-year record of ground-based observations for 1920-1952. Although the SAO data are disputed in reliability (Abbot 2007) and clearly contain a non-solar signal, they are the only available long-term measurements of the TSI in the first half of the twentieth-century. The data show an increase of $1 \pm 0.5(1.5 \pm 0.5) \mathrm{W} / \mathrm{m}^{2}$ per decade for the period 1928-1947 (1920-1952). Because we are aware that maintaining a stable calibration to better than $0.1 \%$ over 30 years is very demanding, we cannot claim that the historical data confirm our reconstruction. Nevertheless, it is intriguing to note how well the SAO trend agrees with our TSI reconstruction.

Our reconstructed solar spectral irradiance comprises spectra from $130 \mathrm{~nm}$ to $10 \mu \mathrm{m}$. Figure 4 presents a reconstruction of the integrated flux for several selected spectral regions. The contrast between different brightness components of the quiet Sun is especially high in the UV, which results in a large historical variability of the UV spectral irradiance. The irradiance in the Schumann-Runge bands and Herzberg continuum increases from the Maunder minimum to the present by about $26.6 \%$ and $10.9 \%$ respectively, which is much larger than $0.4 \%$ for the TSI and the visible region. The variability is also relatively high around the $\mathrm{CN}$ violet system, whose strength is very sensitive to even small temperature differences because of the high value of the dissociation potential. The large UV variability reported here is especially of importance to the climate community because it influences climate via an indirect, non-linearly amplified forcing (Haigh 1994; Egorova et al. 2004).

We are aware that the choice of model A is responsible for a relatively large percentage of the uncertainty in our results. Higher resolution observations have recently become available, and model A could possibly be improved in future studies. We emphasize that model A is not the coldest possible quiet Sun model, and therefore our estimate is not a lower limit of the Sun's energy output. The coldest model would be 
A. I. Shapiro et al.: A new approach to long-term reconstruction of the solar irradiance leads to large historical solar forcing
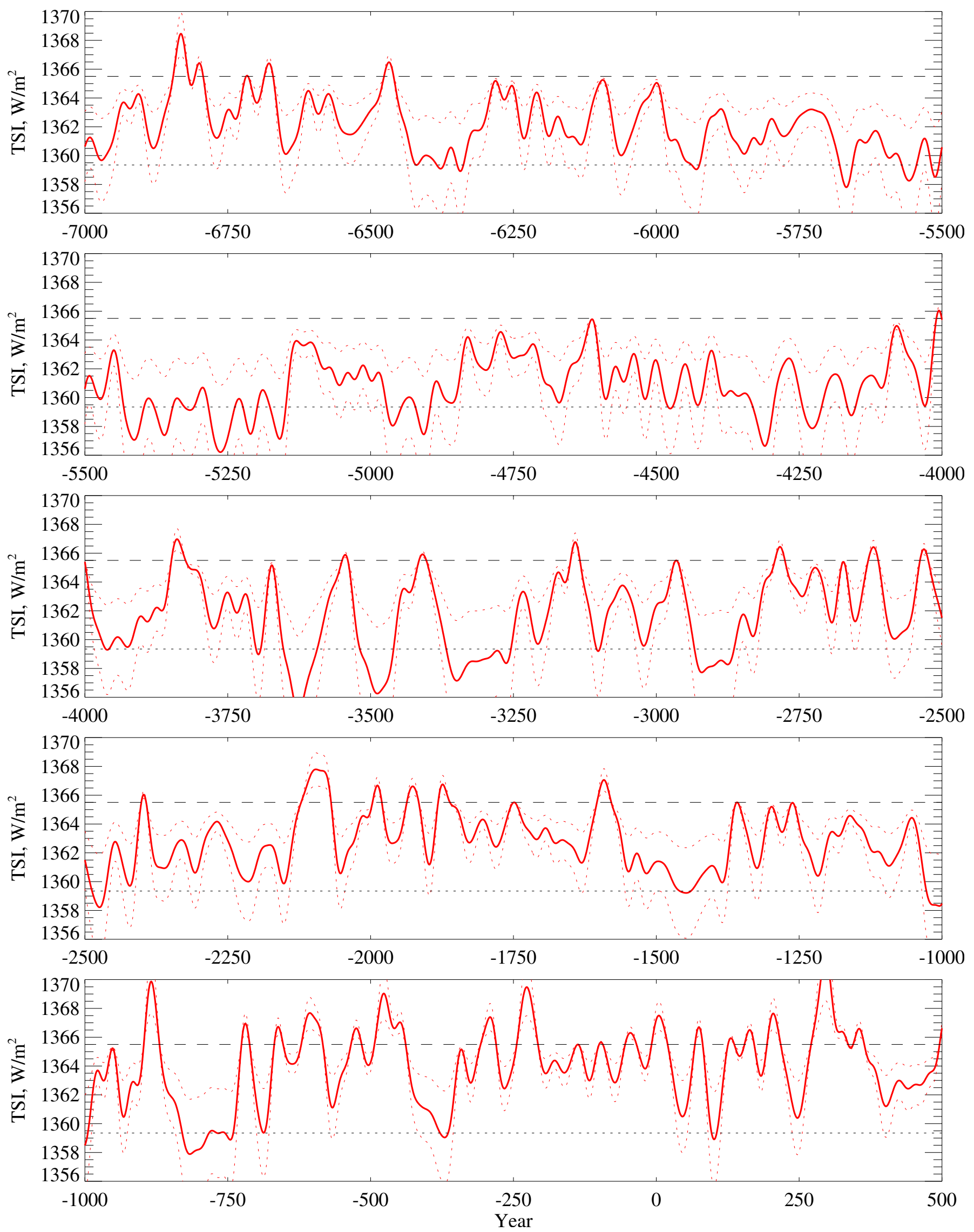

Fig. 3. TSI reconstruction from $7000 \mathrm{BC}$ to $500 \mathrm{AD}$. The reconstruction is based on the modulation potential derived from the ${ }^{10} \mathrm{Be}$ records from the Greenland Ice core Project. Red dotted lines indicate the estimated error bars of the reconstruction. Black dashed and dotted lines indicate the TSI for the 1996 solar minimum and the lowest TSI during the Maunder minimum, respectively. 

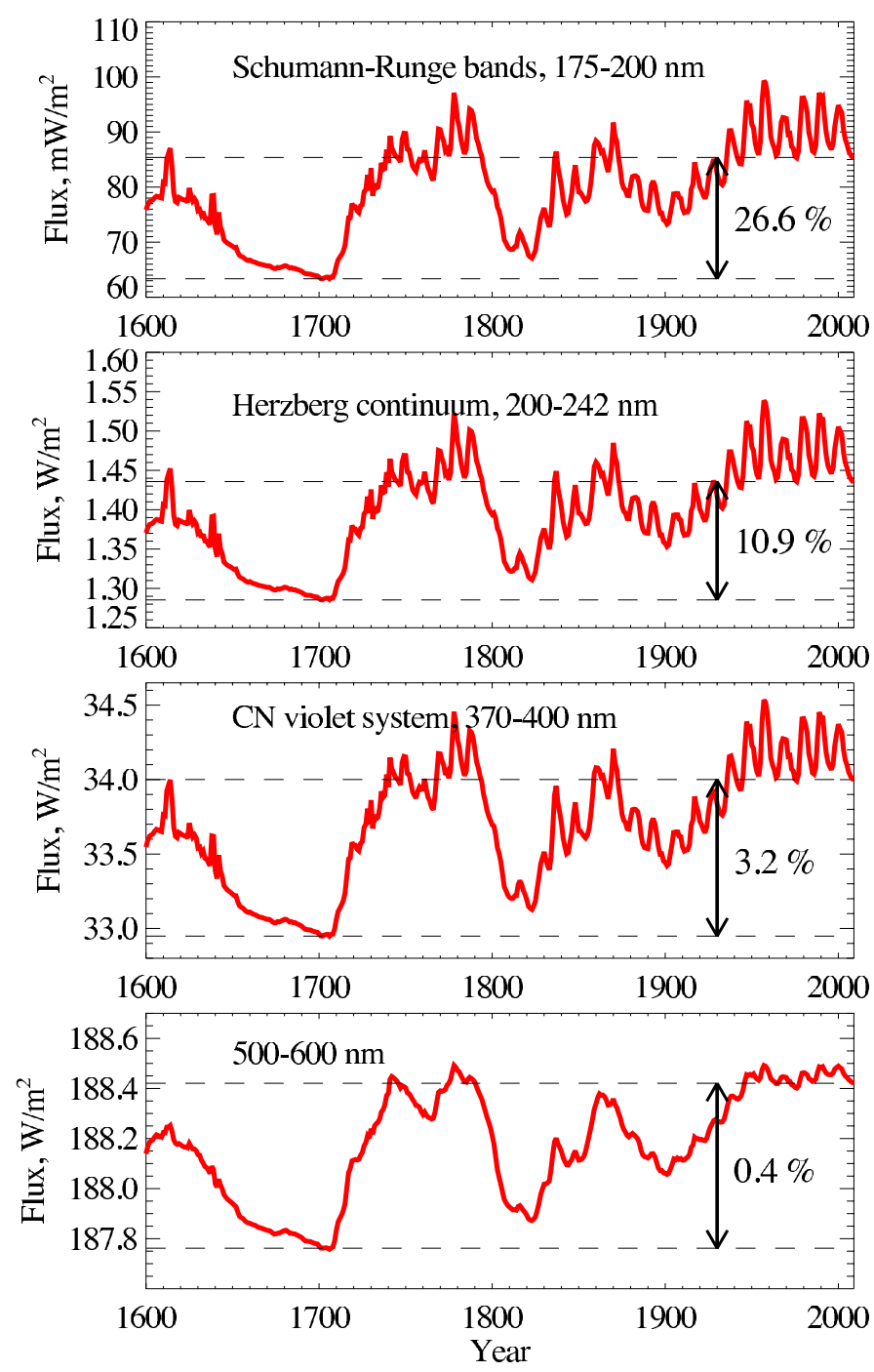

Fig. 4. Reconstruction of the integrated spectral irradiance in selected wavelength bands. This reconstruction is based on the composite of the ${ }^{10} \mathrm{Be}$ South Pole record and neutron monitor data (red curve in Fig. 2). Panels from bottom to top: the 500-600 nm band is representative of the time evolution of the visible irradiance. The $\mathrm{CN}$ violet system is one of the most variable bands that is accessible from the ground. The Herzberg continuum is crucial for ozone production in the stratosphere. The Schumann-Runge band is important for heating processes in the middle atmosphere.

a non-magnetic atmospheric structure without a chromosphere and corona. Because model A contains some remaining magnetic activity, our approach does not imply that the solar dynamo stops during the periods when the modulation potential is equal to zero. Let us also notice that the modulation potential never reached zero for the last 400 years (see Fig. 2).

\section{Conclusions}

We presented a new technique to reconstruct the total and spectral solar irradiance over the Holocene. We obtained a large historical solar forcing between the Maunder minimum and the present, as well as a significant increase in solar irradiance in the first half of the twentieth-century. Our value of the historical solar forcing is remarkably larger than other estimations published in recent literature.

We note that our conclusions cannot be tested on the basis of the last 30 years of solar observations because, according to the proxy data, the Sun was in a maximum plateau state in its long-term evolution. All recently published reconstructions agree well during the satellite observational period and diverge only in the past. This implies that the observational data do not allow to select and favor one of the proposed reconstructions. Therefore, until new evidence becomes available, we are in a situation where different approaches and hypothesis yield different solar forcing values. Our result allows the climate community to evaluate the full range of the present uncertainty in solar forcing.

The full dataset of the solar spectral irradiance back to 7000 $\mathrm{BC}$ is available upon request.

Acknowledgements. We are grateful to Friedhelm Steinhilber and Jürg Beer for useful discussions and help with data. The research leading to this paper was supported by the Swiss National Science Foundation under grant CRSI122-130642 (FUPSOL) and also received funding from European Community's Seventh Framework Programme (FP7/2007-2013) under grant agreement N 218816 (SOTERIA). M.H. appreciates funding from the Swiss Holcim Foundation.

\section{References}

Abbot, C. G. 2007, The Smithsonian Astrophysical Observatory Solar Constant Database

Beer, J., Tobias, S., \& Weiss, N. 1998, Sol. Phys., 181, 237

Smyth, C. P. 1855, MNRAS, 16, 1

Castagnoli, G., \& Lal, D. 1980, Radiocarbon, 22, 133

Charbonneau, P. 2010, Liv. Rev. Sol. Phys., 7, 3

de Wijn, A. G., Stenflo, J. O., Solanki, S. K., \& Tsuneta, S. 2009, Space Sci. Rev., 144, 275

Dewitte, S., Crommelynck, D., Mekaoui, S., \& Joukoff, A. 2004, Sol. Phys., 224, 209

Domingo, V., Ermolli, I., Fox, P., et al. 2009, Space Sci. Rev., 145, 337

Egorova, T., Rozanov, E., Manzini, E., et al. 2004, Geophys. Res. Lett., 31, L06119

Fontenla, J., White, O. R., Fox, P. A., Avrett, E. H., \& Kurucz, R. L. 1999, ApJ, 518,480

Foukal, P., \& Milano, L. 2001, Geophys. Res. Lett., 28, 883

Fröhlich, C. 2006, Space Sci. Rev., 125, 53

Fröhlich, C. 2009, A\&A, 501, L27

Gray, L. J., Beer, J., Geller, M., et al. 2010, Rev. Geophys., 48, 4001

Haberreiter, M., Schmutz, W., \& Hubeny, I. 2008, A\&A, 492, 833

Haigh, J. D. 1994, Nature, 370, 544

Haigh, J. D. 2007, Liv. Rev. Sol. Phys., 4, 2

Hall, J. C., \& Lockwood, G. W. 2004, ApJ, 614, 942

Herbst, K., Kopp, A., Heber, B., et al. 2010, J. Geophys. Res. (Space Physics), 115, D00I20

Hoyt, D. V., \& Schatten, K. H. 1997, The role of the sun in climate change (Oxford University Press), ed. D. V. Hoyt, \& K. H. Schatten, 279

Hoyt, D. V., \& Schatten, K. H. 1998, Sol. Phys., 179, 189

Hoyt, D. V., Kyle, H. L., Hickey, J. R., \& Maschhoff, R. H. 1992, in Solar, Electromagnetic Radiation Study for Solar Cycle 22, SEL NOAA ERL, Boulder, ed. R. F. Donnelly, 43

Kleint, L., Berdyugina, S. V., Shapiro, A. I., \& Bianda, M. 2010, in ASP Conf. Ser. 428, ed. S. R. Cranmer, J. T. Hoeksema, \& J. L. Kohl, 103

Krivova, N. A., \& Solanki, S. K. 2008, J. Astrophys. Astron., 29, 151

Krivova, N. A., Solanki, S. K., Fligge, M., \& Unruh, Y. C. 2003, A\&A, 399, L1

Krivova, N. A., Balmaceda, L., \& Solanki, S. K. 2007, A\&A, 467, 335

Krivova, N. A., Solanki, S. K., \& Wenzler, T. 2009a, Geophys. Res. Lett., 36, L20101

Krivova, N. A., Solanki, S. K., Wenzler, T., \& Podlipnik, B. 2009b, J. Geophys. Res. (Atmospheres), 114, D00I04

Krivova, N. A., Solanki, S. K., \& Unruh, Y. C. 2011, J. Atm. Sol.-Terrestr. Phys., 73,223

Lean, J., Beer, J., \& Bradley, R. 1995, Geophys. Res. Lett., 22, 3195

Lockwood, M. 2010, Proc. Roy. Soc. A, 466, 303

Lockwood, M., Stamper, R., \& Wild, M. N. 1999, Nature, 399, 437 
A. I. Shapiro et al.: A new approach to long-term reconstruction of the solar irradiance leads to large historical solar forcing

McCracken, K. G., McDonald, F. B., Beer, J., Raisbeck, G., \& Yiou, F. 2004, J. Geophys. Res. (Space Physics), 109, 12103

Muscheler, R., Joos, F., Beer, J., et al. 2007, Quat. Sci. Rev., 26, 82

Shapiro, A. I., Schmutz, W., Schoell, M., Haberreiter, M., \& Rozanov, E. 2010, A\&A, 517, A48

Siscoe, G. L. 1978, Nature, 276, 348

Solanki, S. K., \& Krivova, N. A. 2004, Sol. Phys., 224, 197

Solanki, S. K., Schüssler, M., \& Fligge, M. 2000, Nature, 408, 445

Solanki, S. K., Usoskin, I. G., Kromer, B., Schüssler, M., \& Beer, J. 2004, Nature, 431, 1084

Solomon, S., Qin, D., Manning, M., et al. 2007, IPCC, Climate Change 2007:

The Physical Science Basis, Contribution of Working Group I to the Fourth

Assessment Report of the Intergovernmental Panel on Climate Change
Steinhilber, F., Abreu, J. A., \& Beer, J. 2008, Astrophys. Space Sci. Trans., 4, 1 Steinhilber, F., Beer, J., \& Fröhlich, C. 2009, Geophys. Res. Lett., 36, L19704 Stenflo, J. O. 1982, Sol. Phys., 80, 209

Usoskin, I. G. 2008, Liv. Rev. Sol. Phys., 5, 3

Usoskin, I. G., Mursula, K., \& Kovaltsov, G. A. 2001, J. Geophys. Res., 106 16039

Usoskin, I. G., Alanko-Huotari, K., Kovaltsov, G. A., \& Mursula, K. 2005, J. Geophys. Res. (Space Physics), 110, A12108

Vernazza, J. E., Avrett, E. H., \& Loeser, R. 1981, ApJS, 45, 635

Vonmoos, M., Beer, J., \& Muscheler, R. 2006, J. Geophys. Res. (Space Physics), 111, A10105

Wang, Y., Lean, J. L., \& Sheeley, Jr., N. R. 2005, ApJ, 625, 522

Willson, R. C., \& Mordvinov, A. V. 2003, Geophys. Res. Lett., 30, GL016038 


\section{Appendix A: Spectral synthesis}

\section{A.1. Quiet Sun}

The quiet Sun is a combination of different brightness components and the evolution of their fractional contributions drives its activity and long-term irradiance variability. The four main components of the quiet Sun (Vernazza et al. 1981; Fontenla et al. 1999) are: component A (faint supergranule cell interior), component $\mathrm{C}$ (average supergranule cell interior), component $\mathrm{E}$ (average network or quiet network), and component $\mathrm{F}$ (bright network).

We calculated the synthetic spectra $I_{\mathrm{A}}, I_{\mathrm{C}}, I_{\mathrm{E}}, I_{\mathrm{P}}$ of all these components employing the NLTE (non-local thermodynamic equilibrium) COde for Solar Irradiance (Haberreiter et al. 2008; Shapiro et al. 2010) (COSI). Recently, Shapiro et al. (2010) showed that COSI calculations with the atmosphere model for component $\mathrm{C}$ reproduces spectral irradiance measurements from the last two solar minima with good accuracy. This is used in Eq. (1) from the main text, where $I_{\text {present }}(\lambda)$ is substituted by the irradiance $I_{\mathrm{C}}(\lambda)$ for component $\mathrm{C}$.

The evolution of the magnetic activity of the quiet Sun can be represented by the time-dependent fractional contributions (i.e. filling factors) of different components of the quiet Sun. The quiet Sun can be described by model A with varying contributions from the brighter components. We compose the quiet Sun using model $\mathrm{A}$ and model $\mathrm{E}$, which is an adequate representation of the brighter contributions.

Using COSI we demonstrate (see Fig. A.1) that the solar irradiance for model $\mathrm{C}$ and, therefore, the measured solar irradiance for the last two minima, can be successfully reproduced with a combination of $\alpha_{\text {present }}^{\mathrm{A}}=43 \%$ model $\mathrm{A}$ and $\alpha_{\text {present }}^{\mathrm{E}}=57 \%$ model $\mathrm{E}\left(\alpha_{\text {present }}^{\mathrm{A}}+\alpha_{\text {present }}^{\mathrm{E}}=1\right)$ :

$I_{\text {quiet }}^{\text {present }}(\lambda)=I_{\mathrm{C}}(\lambda)=\alpha_{\text {present }}^{\mathrm{A}} I_{\mathrm{A}}(\lambda)+\alpha_{\text {present }}^{\mathrm{E}} I_{\mathrm{E}}(\lambda)$.

The linear scaling of the magnetic activity of the quiet Sun with proxies, as described in the main text, is equivalent to setting the model E filling factor to be proportional to a chosen proxy. Then the time-dependent irradiance of the quiet Sun can be calculated:

$I_{\text {quiet }}(\lambda, t)=\left(1-\alpha^{\mathrm{E}}(t)\right) I_{\mathrm{A}}(\lambda)+\alpha^{\mathrm{E}}(t) I_{\mathrm{E}}(\lambda)$,

where

$\alpha^{\mathrm{E}}(t)=\frac{\operatorname{Proxy}(t)}{\mathcal{P r o x}_{\text {present }}} \alpha_{\text {present }}^{\mathrm{E}}$.

The set of Eqs. (A.1-A.3) is equivalent to Eq. (1).

There is an ongoing discussion of whether the trend in the filling factor of the quiet network can be detected.

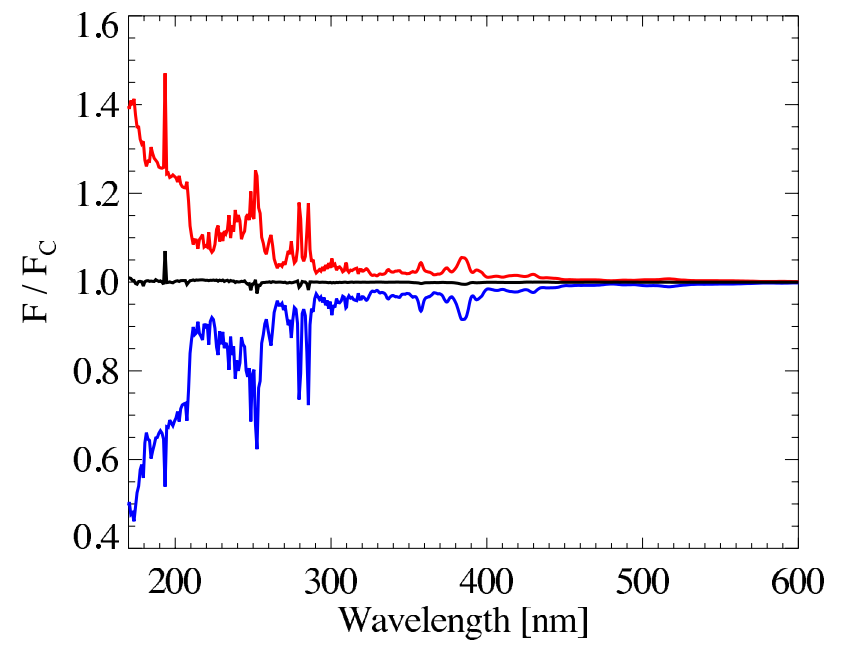

Fig. A.1. Representation of the quiet Sun by a combination of models. Ratios of synthetic solar spectra of models A (blue) and E (red) to model $\mathrm{C}$. The black line results by combining $57 \%$ of the model $\mathrm{E}$ spectrum with $43 \%$ of the model A spectrum. The spectral signatures are almost perfectly cancelled, implying that the combination of both spectra is equal to the model $\mathrm{C}$ spectrum. The latter has been demonstrated to accurately reproduce the observed solar spectra during the last two minima (Shapiro et al. 2010). Hence, a combination of spectra for models A and E reproduces the observed quiet Sun spectrum.

It was suggested (Foukal \& Milano 2001) that the analysis of the historical Mt. Wilson observations exclude the existence of the trend between 1914 and 1996. However, this analysis was criticized for using uncalibrated data (Solanki \& Krivova 2004) and also contradicts other studies (Lockwood 2010). Thus only the future long-term monitoring of the quiet Sun with high resolution or the recently proposed (Kleint et al. 2010) monitoring of weak turbulent magnetic fields can help to clarify this question.

\section{A.2. Active Sun}

From 1610 onward we have additional information from the sunspot number, which allows the calculation of the active regions contribution to the solar irradiance $\left(I_{\text {active }}(\lambda, t)\right.$ in Eq. (2)). For this we follow the approach by Krivova et al. (2003). Because our main goal is to reproduce the centennial solar variability and because magnetograms are unavailable for historical time periods, we scale the faculae and the active network filling factors with the sunspot number instead of using filling factors derived from available magnetogram data. The synthetic spectra are then added according to their filling factors, and the TSI is then determined by integrating over all wavelengths. 